\title{
Alternative representation of the linear canonical integral transform
}

\author{
Tatiana Alieva \\ Facultad de Ciencias Físicas, Universidad Complutense de Madrid, Ciudad Universitaria s/n, Madrid 28040, Spain \\ Martin J. Bastiaans \\ Faculteit Elektrotechniek, Technische Universiteit Eindhoven, Postbus 513, 5600 MB Eindhoven, Netherlands
}

Received June 24, 2005

Starting with the Iwasawa-type decomposition of a first-order optical system (or ABCD system) as a cascade of a lens, a magnifier, and an orthosymplectic system (a system that is both symplectic and orthogonal), a further decomposition of the orthosymplectic system in the form of a separable fractional Fourier transformer embedded between two spatial-coordinate rotators is proposed. The resulting decomposition of the entire first-order optical system then shows a physically attractive representation of the linear canonical integral transformation, which, in contrast to Collins integral, is valid for any ray transformation matrix. (C) 2005 Optical Society of America

OCIS codes: $070.2580,070.4690,080.2730,120.4820$.

Any lossless first-order optical system can be described by its ray transformation matrix, ${ }^{1}$ which relates the position $\mathbf{r}_{i}$ and direction $\mathbf{q}_{i}$ of an incoming ray to the position $\mathbf{r}_{o}$ and direction $\mathbf{q}_{o}$ of the outgoing ray:

$$
\left(\begin{array}{l}
\mathbf{r}_{o} \\
\mathbf{q}_{o}
\end{array}\right)=\left(\begin{array}{ll}
\mathbf{A} & \mathbf{B} \\
\mathbf{C} & \mathbf{D}
\end{array}\right)\left(\begin{array}{l}
\mathbf{r}_{i} \\
\mathbf{q}_{i}
\end{array}\right) \equiv \mathbf{T}\left(\begin{array}{l}
\mathbf{r}_{i} \\
\mathbf{q}_{i}
\end{array}\right)
$$

The ray transformation matrix $\mathbf{T}$ of such a system is real and symplectic, yielding the relations

$$
\begin{array}{lll}
\mathbf{A B}^{t}=\mathbf{B A}^{t}, & \mathbf{C D}^{t}=\mathbf{D C}^{t}, & \mathbf{A D}^{t}-\mathbf{B C}^{t}=\mathbf{I}, \\
\mathbf{A}^{t} \mathbf{C}=\mathbf{C}^{t} \mathbf{A}, & \mathbf{B}^{t} \mathbf{D}=\mathbf{D}^{t} \mathbf{B}, & \mathbf{A}^{t} \mathbf{D}-\mathbf{C}^{t} \mathbf{B}=\mathbf{I} .
\end{array}
$$

Using the submatrices $\mathbf{A}, \mathbf{B}$, and $\mathbf{D}$, and assuming that $\mathbf{B}$ is a nonsingular matrix, we can represent the first-order optical system by the Collins integral ${ }^{2}$

$$
\begin{aligned}
f_{o}\left(\mathbf{r}_{o}\right)= & \frac{\exp (i \phi)}{\sqrt{\operatorname{det} i \mathbf{B}}} \iint_{-\infty}^{\infty} f_{i}\left(\mathbf{r}_{i}\right) \exp \left[i \pi \left(\mathbf{r}_{i}^{t} \mathbf{B}^{-1} \mathbf{A} \mathbf{r}_{i}\right.\right. \\
& \left.\left.-2 \mathbf{r}_{i}^{t} \mathbf{B}^{-1} \mathbf{r}_{o}+\mathbf{r}_{o}^{t} \mathbf{D} \mathbf{B}^{-1} \mathbf{r}_{o}\right)\right] \mathrm{d} \mathbf{r}_{i},
\end{aligned}
$$

in which the output amplitude $f_{o}(\mathbf{r})$ is expressed in terms of the input amplitude $f_{i}(\mathbf{r})$. The phase factor $\exp (i \phi)$ in Eq. (3) has been included for completeness to cope with the optical path length and the metaplectic sign problem; unless absolutely necessary, it will usually be omitted. In the case that $\mathbf{B}$ equals the null matrix, $\mathbf{B}=\mathbf{0}$, Collins integral (3) reduces to

$$
f_{o}(\mathbf{r})=f_{i}\left(\mathbf{A}^{-1} \mathbf{r}\right) \exp \left(i \pi \mathbf{r}^{t} \mathbf{C A}^{-1} \mathbf{r}\right) / \sqrt{|\operatorname{det} \mathbf{A}|} .
$$

The singular case $\operatorname{det} \mathbf{B}=0$ with $\mathbf{B} \neq \mathbf{0}$, however, is rather difficult to handle.

To treat the singular case, Moshinsky and Quesne ${ }^{3}$ showed that any symplectic ABCD matrix with a singular submatrix $\mathbf{B}$ can be decomposed as

$$
\left(\begin{array}{ll}
\mathbf{A} & \mathbf{B} \\
\mathbf{C} & \mathbf{D}
\end{array}\right)=\left(\begin{array}{cc}
\mathbf{I} & \mathbf{B}^{\prime} \\
\mathbf{0} & \mathbf{I}
\end{array}\right)\left(\begin{array}{cc}
\mathbf{A}-\mathbf{B}^{\prime} \mathbf{C} & \mathbf{B}-\mathbf{B}^{\prime} \mathbf{D} \\
\mathbf{C} & \mathbf{D}
\end{array}\right),
$$

in which $\mathbf{B}^{\prime}$ is a nonsingular diagonal matrix and $\operatorname{det}\left(\mathbf{B}-\mathbf{B}^{\prime} \mathbf{D}\right) \neq 0$. After doing so, they could then use the Collins integral (3) for each of the two subsystems in the cascade (5) separately, thus avoiding the singular case. The way to find the diagonal matrix $\mathbf{B}^{\prime}$, however, is not easy.

In this Letter we restrict ourselves to the case that $\mathbf{A}, \mathbf{B}, \mathbf{C}$, and $\mathbf{D}$ are $2 \times 2$ matrices and we propose a representation of the linear canonical integral transformation in an alternative form that is valid for any ray transformation matrix, whether or not it has a singular submatrix B. Our method is based on the Iwasawa decomposition, ${ }^{4,5}$ followed by a further decomposition of an orthosymplectic system into a separable fractional Fourier transformer embedded between two spatial-coordinate rotators.

After properly normalizing to dimensionless variables, any symplectic matrix can be decomposed in the Iwasawa form (Ref. 5, Secs. 9.5 and 10.2)

$$
\left(\begin{array}{ll}
\mathbf{A} & \mathbf{B} \\
\mathbf{C} & \mathbf{D}
\end{array}\right)=\left(\begin{array}{cc}
\mathbf{I} & \mathbf{0} \\
-\mathbf{G} & \mathbf{I}
\end{array}\right)\left(\begin{array}{cc}
\mathbf{S} & \mathbf{0} \\
\mathbf{0} & \mathbf{S}^{-1}
\end{array}\right)\left(\begin{array}{cc}
\mathbf{X} & \mathbf{Y} \\
-\mathbf{Y} & \mathbf{X}
\end{array}\right),
$$

in which the first matrix represents a lens described by the symmetric matrix

$$
\mathbf{G}=-\left(\mathbf{C A}^{t}+\mathbf{D B} \mathbf{B}^{t}\right)\left(\mathbf{A A}^{t}+\mathbf{B B}^{t}\right)^{-1},
$$

the second matrix represents a magnifier described by the symmetric matrix

$$
\mathbf{S}=\left(\mathbf{A} \mathbf{A}^{t}+\mathbf{B} \mathbf{B}^{t}\right)^{1 / 2},
$$

and the third matrix represents a so-called orthosymplectic system - a system that is both orthogonal and symplectic-described by the unitary matrix

$$
\mathbf{U}=\mathbf{X}+i \mathbf{Y}=\left(\mathbf{A A}^{t}+\mathbf{B B}^{t}\right)^{-1 / 2}(\mathbf{A}+i \mathbf{B}) .
$$

$\mathbf{B}=\mathbf{S Y}$, and since $\mathbf{S}$ is nonsingular, a singularity of $\mathbf{B}$ is only due to the orthosymplectic system. 
To treat the orthosymplectic system in more detail, we remark that if $\mathbf{U}_{1}$ and $\mathbf{U}_{2}$ are the unitary representations of two orthosymplectic matrices $\mathbf{T}_{1}$ and $\mathbf{T}_{2}$, then the product $\mathbf{U}_{2} \mathbf{U}_{1}$ is the unitary representation of the cascade $\mathbf{T}_{2} \mathbf{T}_{1}$. The unitary matrix

$$
\mathbf{U}_{r}(\vartheta)=\mathbf{X}_{r}(\vartheta)=\left(\begin{array}{cc}
\cos \vartheta & \sin \vartheta \\
-\sin \vartheta & \cos \vartheta
\end{array}\right)
$$

and its associated orthosymplectic one

$$
\mathbf{T}_{r}(\vartheta)=\left(\begin{array}{cccc}
\cos \vartheta & \sin \vartheta & 0 & 0 \\
-\sin \vartheta & \cos \vartheta & 0 & 0 \\
0 & 0 & \cos \vartheta & \sin \vartheta \\
0 & 0 & -\sin \vartheta & \cos \vartheta
\end{array}\right)
$$

see Ref. 5, Eq. (10.16), correspond to a rotator with rotation angle $\vartheta$, which produces a rotation through the angle $\vartheta$ both for the spatial variables $(x, y)$ and the spatial-frequency variables $\left(q_{x}, q_{y}\right)$. The unitary matrix

$\mathbf{U}_{f}\left(\gamma_{x}, \gamma_{y}\right)=\mathbf{X}_{f}\left(\gamma_{x}, \gamma_{y}\right)+i \mathbf{Y}_{f}\left(\gamma_{x}, \gamma_{y}\right)=\left(\begin{array}{cc}\exp \left(i \gamma_{x}\right) & 0 \\ 0 & \exp \left(i \gamma_{y}\right)\end{array}\right)$

and its associated orthosymplectic one

$\mathbf{T}_{f}\left(\gamma_{x}, \gamma_{y}\right)=\left(\begin{array}{cccc}\cos \gamma_{x} & 0 & \sin \gamma_{x} & 0 \\ 0 & \cos \gamma_{y} & 0 & \sin \gamma_{y} \\ -\sin \gamma_{x} & 0 & \cos \gamma_{x} & 0 \\ 0 & -\sin \gamma_{y} & 0 & \cos \gamma_{y}\end{array}\right)$,

cf. Ref. 5, Eq. (10.31), correspond to a separable fractional Fourier transformer with fractional angles $\gamma_{x}$ and $\gamma_{y}$ in the $x$ and the $y$ directions, respectively, which produces a rotation through $\gamma_{x}$ for the spacespatial-frequency combination $\left(x, q_{x}\right)$ and a rotation through $\gamma_{y}$ for the space-spatial-frequency combination $\left(y, q_{y}\right)$. In the one-dimensional case, the orthosymplectic system that appears in the Iwasawa decomposition (6) is described by the scalar $u=(a+i b)$ $\times\left(a^{2}+b^{2}\right)^{-1 / 2}=\exp (i \gamma)$, cf. (9), corresponding to a (onedimensional) fractional Fourier transformer; see also Ref. 6, Section 9.7, in particular Eqs. (9.124)-(9.128).

We now show that any unitary matrix $\mathbf{U}$ can be represented as a separable fractional Fourier transformer $\mathbf{U}_{f}\left(\gamma_{x}, \gamma_{y}\right)$ embedded in between two rotators $\mathbf{U}_{r}(\alpha)$ and $\mathbf{U}_{r}(\beta)$, cf. Ref. 5, Eq. (10.32):

$$
\mathbf{U}=\mathbf{U}_{r}(\beta) \mathbf{U}_{f}\left(\gamma_{x}, \gamma_{y}\right) \mathbf{U}_{r}(\alpha) .
$$

Note that $\operatorname{det} \mathbf{U}_{f}\left(\gamma_{x}, \gamma_{y}\right)=\exp \left[i\left(\gamma_{x}+\gamma_{y}\right)\right]$ and that $\operatorname{det} \mathbf{U}_{r}(\vartheta)=1$. We will demonstrate how the four angles $\alpha, \beta, \gamma_{x}$, and $\gamma_{y}$ follow from the entries of the matrix $\mathbf{U}=\mathbf{X}+i \mathbf{Y}$ :

$$
\begin{aligned}
& X_{11}=\cos \alpha \cos \beta \cos \gamma_{x}-\sin \alpha \sin \beta \cos \gamma_{y}, \\
& X_{12}=\sin \alpha \cos \beta \cos \gamma_{x}+\cos \alpha \sin \beta \cos \gamma_{y}, \\
& X_{21}=-\cos \alpha \sin \beta \cos \gamma_{x}-\sin \alpha \cos \beta \cos \gamma_{y},
\end{aligned}
$$

$$
\begin{aligned}
& X_{22}=-\sin \alpha \sin \beta \cos \gamma_{x}+\cos \alpha \cos \beta \cos \gamma_{y}, \\
& Y_{11}=\cos \alpha \cos \beta \sin \gamma_{x}-\sin \alpha \sin \beta \sin \gamma_{y}, \\
& Y_{12}=\sin \alpha \cos \beta \sin \gamma_{x}+\cos \alpha \sin \beta \sin \gamma_{y}, \\
& Y_{21}=-\cos \alpha \sin \beta \sin \gamma_{x}-\sin \alpha \cos \beta \sin \gamma_{y}, \\
& Y_{22}=-\sin \alpha \sin \beta \sin \gamma_{x}+\cos \alpha \cos \beta \sin \gamma_{y} .
\end{aligned}
$$

We note the relations

$$
\begin{gathered}
\mathbf{U}_{r}(\vartheta+\pi)=-\mathbf{U}_{r}(\vartheta), \\
\mathbf{U}_{f}\left(\gamma_{x}+\pi, \gamma_{y}+\pi\right)=-\mathbf{U}_{f}\left(\gamma_{x}, \gamma_{y}\right),
\end{gathered}
$$

from which we conclude that in the cascade (14) the fractional angles $\gamma_{x}$ and $\gamma_{y}$ may be safely restricted to the interval $[0, \pi)$, if at the same time we allow at least one of the rotation angles $\alpha$ and $\beta$ to be in a full interval of length $2 \pi$. Moreover, from the relation

$$
\mathbf{U}_{r}(\pi / 2) \mathbf{U}_{f}\left(\gamma_{x}, \gamma_{y}\right) \mathbf{U}_{r}(-\pi / 2)=\mathbf{U}_{f}\left(\gamma_{y}, \gamma_{x}\right)
$$

we conclude that we can always interchange the fractional angles; in other words, we may freely choose the fractional angle $\gamma_{x}$ to be the largest. Without loss of generality we may thus choose $0 \leqslant \gamma_{y} \leqslant \gamma_{x}<\pi$.

From the two relations

$$
\begin{aligned}
& \cos \left(\gamma_{x}+\gamma_{y}\right)=\operatorname{Re}\{\operatorname{det} \mathbf{U}\}, \\
& \sin \left(\gamma_{x}+\gamma_{y}\right)=\operatorname{Im}\{\operatorname{det} \mathbf{U}\},
\end{aligned}
$$

we can determine the sum $\gamma_{x}+\gamma_{y}=2 \gamma_{1}$ (with $\gamma_{1}$ in the interval $0 \leqslant \gamma_{1}<\pi$ ), and from the relation

$$
\operatorname{det} \mathbf{X}+\operatorname{det} \mathbf{Y}=\cos \left(\gamma_{x}-\gamma_{y}\right)
$$

we determine the difference $\gamma_{x}-\gamma_{y}=2 \gamma_{2}$ (with $\gamma_{2}$ in the interval $0 \leqslant \gamma_{2}<\pi / 2$ ). The two fractional angles $\gamma_{x}$ and $\gamma_{y}$ are thus completely defined.

To determine the rotation angles $\alpha$ and $\beta$, we use the relations

$$
\begin{gathered}
X_{11}+X_{22}-Y_{12}+Y_{21}=2 \cos \left(\alpha+\beta+\gamma_{1}\right) \cos \gamma_{2} \\
X_{12}-X_{21}+Y_{11}+Y_{22}=2 \sin \left(\alpha+\beta+\gamma_{1}\right) \cos \gamma_{2}, \\
-X_{11}+X_{22}+Y_{12}+Y_{21}=2 \sin \left(\alpha-\beta+\gamma_{1}\right) \sin \gamma_{2}, \\
X_{12}+X_{21}+Y_{11}-Y_{22}=2 \cos \left(\alpha-\beta+\gamma_{1}\right) \sin \gamma_{2} .
\end{gathered}
$$

Note that the case $\gamma_{x}=\gamma_{y}$, and thus $\gamma_{2}=0$, is special. In this case we can only determine the sum $\alpha+\beta$ of the two rotation angles. This is obvious, since the cascade (14) now consists of two rotators and an isotropic fractional Fourier transformer with a scalar matrix $\mathbf{U}\left(\gamma_{x}, \gamma_{x}\right)=\exp \left(i \gamma_{x}\right) \mathbf{I} ;$ and since the three 
subsystems commute, we may distribute the total rotation angle $\alpha+\beta$ arbitrarily over the two rotators.

For example, consider the nontrivial singular case $\gamma_{y}=0, \gamma_{x} \neq 0$, for which $\operatorname{det} \mathbf{B}=0$ but $\mathbf{B} \neq \mathbf{0}$, and assume that $Y_{11}, Y_{12}, Y_{21}$, and $Y_{22}$ do not vanish. From Eq. (15) we conclude directly that $Y_{12} / Y_{11}=Y_{22} / Y_{21}$ $=\tan \alpha$ and $Y_{21} / Y_{11}=Y_{22} / Y_{12}=-\tan \beta$. Recall that at least one rotation angle should be in a full interval of length $2 \pi$; choosing rather arbitrarily $\alpha \in[0, \pi)$ and $\beta \in[0,2 \pi)$, the angles then appear in the quadrants as shown in Table 1. The table is also helpful to solve the apparent $\pi$ ambiguity for $\beta$ in the case of vanishing $Y$ values: for instance, to choose between 0 and $\pi$ if $Y_{21}=Y_{22}=0$ (using $Y_{11}$ and $Y_{12}$ ), and between $\pi / 2$ and $3 \pi / 2$ if $Y_{11}=Y_{12}=0$ (using $Y_{21}$ and $Y_{22}$ ).

We conclude that any orthosymplectic system can be realized as a separable fractional Fourier transformer $\mathbf{U}_{f}\left(\gamma_{x}, \gamma_{y}\right)$ embedded between two rotators $\mathbf{U}_{r}(\alpha)$ and $\mathbf{U}_{r}(\beta)$; see Eq. (14).

Using Eqs. (6) and (14), the entire system can finally be decomposed as the cascade

$$
\begin{aligned}
\left(\begin{array}{ll}
\mathbf{A} & \mathbf{B} \\
\mathbf{C} & \mathbf{D}
\end{array}\right)= & \left(\begin{array}{cc}
\mathbf{I} & 0 \\
-\mathbf{G} & \mathbf{I}
\end{array}\right)\left(\begin{array}{cc}
\mathbf{S} & \mathbf{0} \\
\mathbf{0} & \mathbf{S}^{-1}
\end{array}\right)\left(\begin{array}{cc}
\mathbf{X}_{r}(\beta) & \mathbf{0} \\
\mathbf{0} & \mathbf{X}_{r}(\beta)
\end{array}\right) \\
& \times\left(\begin{array}{cc}
\mathbf{X}_{f}\left(\gamma_{x}, \gamma_{y}\right) & \mathbf{Y}_{f}\left(\gamma_{x}, \gamma_{y}\right) \\
-\mathbf{Y}_{f}\left(\gamma_{x}, \gamma_{y}\right) & \mathbf{X}_{f}\left(\gamma_{x}, \gamma_{y}\right)
\end{array}\right)\left(\begin{array}{cc}
\mathbf{X}_{r}(\alpha) & \mathbf{0} \\
\mathbf{0} & \mathbf{X}_{r}(\alpha)
\end{array}\right) .
\end{aligned}
$$

The submatrix $\mathbf{B}$ then takes the form

$$
\mathbf{B}=\mathbf{S X}_{r}(\beta) \mathbf{Y}_{f}\left(\gamma_{x}, \gamma_{y}\right) \mathbf{X}_{r}(\alpha),
$$

and since $\mathbf{S}$ and $\mathbf{X}_{\mathbf{r}}$ are nonsingular, the case $\operatorname{det} \mathbf{B}$ $=0$ arises only for $\operatorname{det} \mathbf{Y}_{f}\left(\gamma_{x}, \gamma_{y}\right)=0$, i.e., for $\sin \gamma_{x} \sin \gamma_{y}=0$, and thus for $\gamma_{y}=0$.

The decomposition (22) has a clear physical interpretation. The cascade starts with a rotator $\mathbf{U}_{r}(\alpha)$ that rotates the coordinate system such that the new axes coincide with the axes of the separable fractional Fourier transformer $\mathbf{U}_{f}\left(\gamma_{x}, \gamma_{y}\right)$. Hence if $f_{\gamma_{x}}\left(x_{o}\right)$ $=\mathcal{R}^{\gamma_{x}}\left[f\left(x_{i}\right)\right]\left(x_{o}\right)$ denotes a one-dimensional fractional Fourier transformation ${ }^{6-8}$ with fractional angle $\gamma_{x}$ (and $0 \leqslant \gamma_{x}<\pi$ ),

Table 1. Quadrants for $\alpha \in[0, \pi)$ and $\beta \in[0,2 \pi)$, for Different Values of the Signs of $Y_{11}, Y_{12}, Y_{21}$, and $Y_{22}$

\begin{tabular}{cccccc}
\hline$Y_{11}$ & $Y_{12}$ & $Y_{21}$ & $Y_{22}$ & $\alpha$ & $\beta$ \\
\hline+ & + & - & - & {$[0, \pi / 2)$} & {$[0, \pi / 2)$} \\
- & + & + & - & {$[\pi / 2, \pi)$} & {$[0, \pi / 2)$} \\
- & - & - & - & {$[0, \pi / 2)$} & {$[\pi / 2, \pi)$} \\
+ & - & + & - & {$[\pi / 2, \pi)$} & {$[\pi / 2, \pi)$} \\
- & - & + & + & {$[0, \pi / 2)$} & {$[\pi, 3 \pi / 2)$} \\
+ & - & - & + & {$[\pi / 2, \pi)$} & {$[\pi, 3 \pi / 2)$} \\
+ & + & + & + & {$[0, \pi / 2)$} & {$[3 \pi / 2,2 \pi)$} \\
- & + & - & + & {$[\pi / 2, \pi)$} & {$[3 \pi / 2,2 \pi)$} \\
\hline
\end{tabular}

$$
\begin{aligned}
& \mathcal{R}^{\gamma_{x}}\left[f\left(x_{i}\right)\right]\left(x_{o}\right) \\
& =\left\{\begin{array}{l}
\frac{\exp \left(i \gamma_{x} / 2\right)}{\sqrt{i \sin \gamma_{x}}} \int_{-\infty}^{\infty} \exp \left[i \pi \frac{\left(x_{o}^{2}+x_{i}^{2}\right) \cos \gamma_{x}}{\sin \gamma_{x}}\right] \\
\times \exp \left[-i 2 \pi \frac{x_{o} x_{i}}{\sin \gamma_{x}}\right] f\left(x_{i}\right) \mathrm{d} x_{i} \quad\left(\gamma_{x} \neq 0\right), \\
f\left(x_{o}\right) \quad\left(\gamma_{x}=0\right),
\end{array}\right.
\end{aligned}
$$

and analogously $f_{\gamma_{x}, \gamma_{y}}\left(\mathbf{r}_{o}\right)=\mathcal{R} \gamma_{x}, \gamma_{y}\left[f\left(\mathbf{r}_{i}\right)\right]\left(\mathbf{r}_{o}\right)$, its twodimensional separable version with angles $\gamma_{x}$ and $\gamma_{y}$, then the input-output relationship for the nonseparable fractional Fourier transformer $\mathbf{U}_{f}\left(\gamma_{x}, \gamma_{y}\right) \mathbf{U}_{r}(\alpha)$ reads $f_{\gamma_{x}, \gamma_{y}}\left(\mathbf{r}_{o}\right)=\mathcal{R}^{\gamma_{x}, \gamma_{y}}\left[f\left(\mathbf{X}_{r}(\alpha) \mathbf{r}_{i}\right)\right]\left(\mathbf{r}_{o}\right)$, or in detail,

$$
\begin{aligned}
f_{\gamma_{x}, \gamma_{y}}\left(x_{o}, y_{o}\right)= & \mathcal{R}^{\gamma_{x}, \gamma_{y}}\left[f \left(x_{i} \cos \alpha+y_{i} \sin \alpha,\right.\right. \\
& \left.\left.-x_{i} \sin \alpha+y_{i} \cos \alpha\right)\right]\left(x_{o}, y_{o}\right) .
\end{aligned}
$$

The separable fractional Fourier transformer $\mathbf{U}_{f}\left(\gamma_{x}, \gamma_{y}\right)$ itself is responsible for a possible degeneration of the submatrix $\mathbf{B}$, but such a degeneration has a clear interpretation: it simply means that for one coordinate (or maybe even for both coordinates) the fractional Fourier transformer acts as an identity system. The cascade then continues with the rotator $\mathbf{U}_{r}(\beta)$, followed by the magnifier $\mathbf{S}$ and the lens $\mathbf{G}$, and brings us to the final input-output relationship for the cascade (22):

$$
\begin{aligned}
f_{o}\left(\mathbf{r}_{o}\right)= & \exp \left(-i \pi \mathbf{r}_{o}^{t} \mathbf{G} \mathbf{r}_{o}\right) / \sqrt{\operatorname{det} \mathbf{S}} \\
& \times \mathcal{R}^{\gamma_{x}, \gamma_{y}}\left[f_{i}\left(\mathbf{X}_{r}(\alpha) \mathbf{r}_{i}\right)\right]\left(\mathbf{X}_{r}(-\beta) \mathbf{S}^{-1} \mathbf{r}_{o}\right) .
\end{aligned}
$$

Equation (26) provides the alternative representation of the linear canonical integral transformation in first-order optical systems, valid for any values of the ray transformation matrix. Note that this equation can also be used for numerical calculation of the canonical transform, using the algorithms developed for the fractional Fourier transformation. ${ }^{6}$

The Spanish Ministry of Education and Science is acknowledged for financial support through a "Ramon y Cajal" grant and projects TIC 2002-01846 (T. Alieva, talieva@fis.ucm.es) and SAB2004-0018 (M. J. Bastiaans, m.j.bastiaans@tue.nl).

\section{References}

1. R. K. Luneburg, Mathematical Theory of Optics (University of California Press, 1966).

2. S. A. Collins, Jr., J. Opt. Soc. Am. 60, 1168 (1970).

3. M. Moshinsky and C. Quesne, J. Math. Phys. 12, 1772 (1971).

4. R. Simon and N. Mukunda, J. Opt. Soc. Am. A 15, 2146 (1998).

5. K. B. Wolf, Geometric Optics on Phase Space (Springer, 2004).

6. H. M. Ozaktas, Z. Zalevsky, and M. A. Kutay, The Fractional Fourier Transform with Applications in Optics and Signal Processing (Wiley, 2001).

7. V. Namias, J. Inst. Math. Appl. 25, 241 (1980).

8. A. C. McBride and F. H. Kerr, IMA J. Appl. Math. 39, 159 (1987). 